\title{
Optimal Compatibility in Systems Markets ${ }^{\text {tr }}$
}

\author{
Sang-Hyun Kim ${ }^{\mathrm{a}}$, Jay Pil Choi ${ }^{\mathrm{b}, *}$ \\ ${ }^{a}$ School of Economics and the ESRC Centre for Competition Policy, University of East Anglia, \\ $U K$ \\ ${ }^{b}$ Department of Economics, Michigan State University, USA and School of Economics, Yonsei \\ University, Korea.
}

\begin{abstract}
We investigate private and social incentives for standardization to ensure marketwide system compatibility in a two-dimensional spatial competition model. We develop a new methodology to analyze competition on a torus and show that there is a fundamental conflict of interest between consumers and producers over the standardization decision. Consumers prefer standardization with full compatibility because it offers more variety that confers a better match with their ideal specifications. However, firms are likely to choose the minimal compatibility to maximize product differentiation and soften competition. This is in sharp contrast to the previous literature that shows the alignment of private and social incentives for compatibility.

JEL Classification: D43, L13, L22
\end{abstract}

Keywords: Compatibility, System Competition, Standardization, Spatial Competition Model

\section{Introduction}

This paper reexamines the incentives for firms to achieve standardization that ensures market-wide system compatibility in a two-dimensional spatial competition

\footnotetext{
ॠWe thank participants in various conferences and seminars for helpful comments, and Michael Cross for editorial assistance. We are also grateful to two anonymous referees and the Advisory Editor for constructive comments which greatly improved the paper. All errors, if any, are ours. This work was supported by the National Research Foundation of Korea Grant funded by the Korean Government (NRF-2014-11-0404) and under Australian Research Council's Discovery Projects funding scheme (project number DP140100007).

${ }^{*}$ Corresponding author Choi)

Email addresses: sang-hyun.kim@uea.ac.uk (Sang-Hyun Kim), choijay@msu.edu (Jay Pil
} 
model. To analyze such an issue, we consider a systems market comprised of two complementary products to be used on a one-to-one basis. We construct a "torus" model to represent two-dimensional spatial competition. The torus model allows characterization of a symmetric equilibrium with more than two firms, as the circular city model does in a one dimensional product space. We develop a novel approach to analyze competition on a torus and show that there is a fundamental conflict of interest between consumers and producers over the standardization decision. Consumers prefer standardization with full compatibility because it offers more variety that confers a better match with their ideal specifications. However, firms are likely to choose the minimal compatibility to maximize product differentiation and soften competition. This is in sharp contrast to the previous literature that shows the alignment of private and social incentives for compatibility (Economides, 1989; Matutes and Regibeau, 1988).

Consider a situation in which all firms in the market are integrated in the sense that they produce both component products. One important decision for them is whether or not to make their components compatible with those of their rivals. In the case of the home audio industry, for instance, full industry-wide compatibility prevails, which allows consumers to combine a receiver of any brand with speakers of any other brands. In the smartphone industry, however, we have incompatibility in the sense that any "apps" developed for the Android operating system cannot be used for any phone based on iOS, the operating system developed by Apple, and vice versa. ${ }^{1}$

The issue of compatibility choice has been studied extensively. The literature on this has addressed two main questions: Do firms have incentives to achieve compatibility across components made by different producers? Is the market compatibility choice socially optimal? Major contributors on this subject, in particular Matutes and Regibeau (1988) and Economides (1989), answered positively to both questions, demonstrating the alignment of private and social incentives for compatibility in the absence of network effects. In particular, Matutes and Regibeau (1988) consider a two-stage game in which two fully integrated firms make their compatibility decisions prior to competition in prices. They show that the equilibrium entails

\footnotetext{
${ }^{1}$ System markets are often characterized by indirect network effects or inter-group network externalities. Implications of network effects in this set-up has been extensively analyzed in the framework of two-sided markets (see, for instance, Armstrong, 2006 and Rochet and Tirole, 2006). In this paper, we analyze incentives to maintain product compatibility in the absence of network externalities to isolate the effects of compatibility on competitive pricing. This also facilitates comparison of our results to the existing literature pioneered by Matutes and Regibeau (1988) and Economides (1989).
} 
full compatibility between component products of the two rival firms because compatibility leads to higher prices than incompatibility. Compatibility also leads to higher social welfare due to the increased variety of systems available. Economides (1989) extends Matutes and Regibeau's analysis by considering a more general case of $n(\geq 2)$ firms. He reconfirms that compatibility prevails in equilibrium, because compatibility leads to higher prices and profits than incompatibility. However, as we explain below, Economides' conclusion is based on an inconsistent analysis, which we intend to rectify in this paper. To this end, we develop a torus model that allows more than two firms to be located symmetrically. We demonstrate that Matutes and Regibeau's results are limited to the special case of two firms and not robust to changes in the number of firms, overturning the conclusion of Economides (1989).

To analyze the desirability of compatibility across different producers of each component products, we adopt the framework of Matutes and Regibeau (1988) to facilitate the comparison of results. However, we modify it to maintain symmetry across system products in a more general case with more than 2 varieties for each component. More precisely, we consider a system good market which consists of two differentiated component goods, called $A$ and $B$, such as hardware and software. ${ }^{2}$ We search for symmetric equilibria with $n$ producers in each of the two differentiated component-markets. The distribution of consumers' preference is modeled as a uniform distribution on a torus, the Cartesian product of two circles.

With a general number of firms in the market, we need to deal with a large number of compatibility possibilities across the two components and location choices for each system variety. In this paper, we focus on two polar cases. Compatibility is the case where any component in market $A$ is compatible with any component in market $B$. For instance, this may be an outcome of industry-wide standardization efforts that ensures interoperability between any components that adhere to the industry standard. The other case we consider is the minimum level of compatibility in which one particular variety of component $A$ is compatible with only one variety of component $B$, and vice versa. We call this regime incompatibility under which no mix-and-match is possible. We consider two alternative market structures, one in which all firms are vertically integrated multi-product firms that produce both components, and one in which each firm is vertically separated and produces only one component. In the integrated firm case, incompatibility would arise if each firm produces a closed system. In the vertically separated firm case, this would arise if each firm has a proprietary technology and firms in market $A$ form an exclusive partnership with partner firms in market $B$.

\footnotetext{
${ }^{2}$ See Katz and Shapiro (1994) for a discussion of economic issues in systems markets.
} 
We show that for all $n \geq 4$, firms prefer incompatibility to compatibility in the integrated firm case (whereas the same holds for $n \geq 3$ in the vertically separated firm case). ${ }^{3}$ In contrast, social welfare is maximized under compatibility. This implies that the alignment of private and social incentives towards compatibility in Matutes and Regibeau is a special result that applies to only $n=2$, and is not robust to changes in the number of firms.

The logic behind our results is simple and can be explained in geometric terms. First, under compatibility there are $n^{2}$ systems available while there are only $n$ systems available under incompatibility. As a result, it is more costly for an individual consumer to change his choice under incompatibility because the second best alternative tends to be farther away from his ideal specification than in the compatibility regime. Second, the measure of marginal consumers (those who are indifferent between two alternatives) is usually smaller under incompatibility. This implies that the marginal gains from a price cut, or equivalently the firms' price cutting incentives, are smaller. Taken together, firms have incentives to produce closed systems or engage in exclusive partnerships that result in incompatibility in order to reduce the intensity of price competition. In particular, we make a novel methodological innovation that provides a clean characterization of the dependence of price competition on the location of competing products in a two-dimensional product space. We show that the intensity of price competition in the market is completely characterized by and is inversely related to a simple parameter called the $m$-to- $d$ ratio, which is a ratio of the measure of marginal consumers to the extent of product differentiation. This parameter operationalizes the notion of the principle of maximum differentiation in a two dimensional product space. On the other hand, as is wellunderstood in the literature, compatibility is desirable for the consumers and for the whole economy since it reduces consumers" "transportation costs" by increasing the variety of systems available in the market.

It is worth mentioning that Economides (1989) analyzed essentially the same problem we are considering in this paper. His analysis, however, was logically inconsistent by implicitly considering two topologically different manifolds to represent the same market - sphere and torus - when he compared two different regimes. ${ }^{4}$ More specifically, he assumed on one hand that consumers are uniformly distributed

\footnotetext{
${ }^{3}$ Monroe (1993) shows by example that the comparison of industry profits across regimes is ambiguous for $n>4$ and depends on firms' locations in product space, but does not provide a systematic analysis as we do.

${ }^{4}$ We are not the first one to notice this error. Matutues and Regibeau (1991) and Farrell, Monroe, and Saloner (1998) also point out the error, but do not pursue the matter further.
} 
on a surface of a sphere that has a great circle of length 1 (p.1167). On the other hand, he represented the market on a two-dimensional plane (see Figure 1 in p.1169 of the paper) which is topologically equivalent (or homeomorphic) to a torus (Cartesian product of two circles) but not to a sphere. ${ }^{5}$ The analysis of the equilibrium under compatibility (Section II) was based on the assumption that the market can be represented as the two-dimensional plane. In the analysis of the equilibrium under incompatibility (Section III), however, he assumed a sphere market: whereas the distance between any two neighboring systems appears to be $\sqrt{2} d$ in the twodimensional plane (Figure 1), that distance is taken as $d$ (as opposed to $\sqrt{2} d$ ) in the analysis in Section III. This would be the case on the surface of the sphere where the length of a diagonal circle is the same with that of a horizontal circle or a vertical circle. But that is not the case in the plane representation.

Even if we ignored this technical problem, Economides's analysis would still appear problematic, because as shown in this paper, how the products are located on the characteristics space matters. Without an inquiry of whether the equilibrium profits would depend on the locations of the products, he prematurely assumed that the system goods are located on the diagonal. The comparison under this locational formation of the products may not be fair. For the compatibility case there is no other formation that enhances the equilibrium profits, but for the incompatibility case there is. As a result, he mistakenly concluded that the Matutes and Regibeau result extends for the general $n$ firm case, with private and social incentives aligned. In contrast, we show in this paper that firms may prefer incompatibility, because under incompatibility they can increase profits by differentiating their products from others.

Our paper mainly contributes to the literature on spatial competition models of compatibility, but also relates to other branches of research including products bundling (e.g. Chen, 1997; Choi, 1996; Denicolo, 2000; Matutes and Regibeau, 1992; Nalebuff, 2004), exclusive dealing (e.g. Besanko and Perry, 1994) and vertical organization of industry in general (Farrell et al., 1998). In our analysis, we consider two alternative market structures. One is where all firms are integrated in the sense that they produce both components of the system. The other case is where all firms produce only one component. Depending on the market structure, our model can also be reinterpreted as an analysis of the incentives for firms to engage in bundling or exclusive dealing arrangements. In the integrated firm case, the effect of incompatibility is equivalent to that of bundling in that consumers are forced

\footnotetext{
${ }^{5}$ For an explanation about homeomorphic relations among two-dimensional manifolds, we refer interested readers to Massey (1991).
} 
to buy the whole system from the same vendor. In the one-component producer case, incompatibility is equivalent to exclusive dealing between a pair of partner firms. For instance, if we reinterpret two component products as a final good and retailing service, respectively, our model can be considered as an analysis of incentives for exclusive dealing contracts. In this context, our paper also provides a useful framework to analyze incentives to engage in bundling decisions or exclusive dealing arrangements in system markets.

Finally, our paper is closely related to Zhou (2014) who analyzes incentives for bundling with an arbitrary number of firms as in our paper. He shows that under fairly general conditions, bundling raises market prices compared to separate sales when the number of firms exceeds a threshold level. Bundling in his model is equivalent to the regime of incompatibility in our model with vertically integrated firms, whereas separate sales correspond to the regime of compatibility. His result is thus consistent with ours even though it was derived from a very different framework. More specifically, he adopts the random utility framework of Perloff and Salop (1985) to model product differentiation in contrast to our spatial competition model. This demonstrates that our main result is robust and not model-specific.

The rest of the paper is organized as follows. In section 2, we present the model of differentiated system products in a toroidal two dimensional product space. In section 3, we first consider integrated firms that produce both components. We analyze market equilibria in two regimes, incompatibility without any mix-and-match possibility, and full compatibility enabled by market-wide standardization. We derive the market equilibrium in each regime and analyze incentives to achieve standardization in the market. We show that under full compatibility, the market equilibrium in each component market can be analyzed in isolation of the other component market. As a result, the market equilibrium replicates the one in the classical circular city model. We also characterize the equilibrium of price competition under any symmetric configuration of product differentiation, which allows the comparison of equilibrium profits under compatibility and under incompatibility. The analysis reveals a fundamental conflict of interest between consumers and producers over the standardization decision. Section 4 contains a discussion about an alternative market structure in which each firm produces only one component as a robustness check of our main results. Concluding remarks follow.

\section{The Model}

Consider a market for system goods that consist of two component products, $A$ and $B$. We assume that these two component products can generate value only 


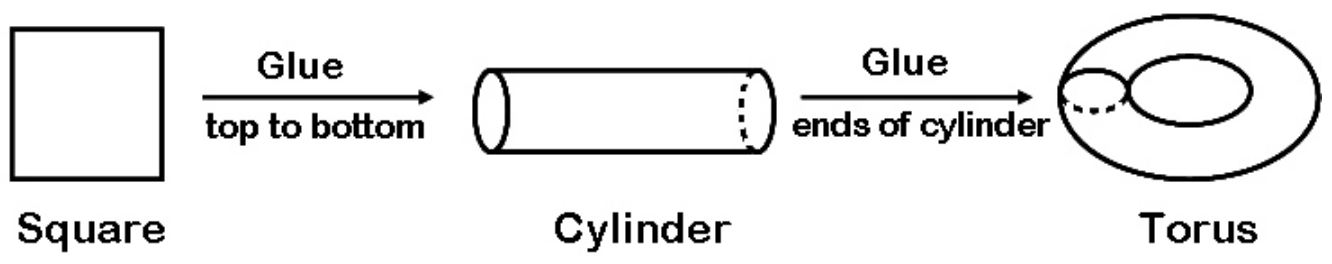

Figure 1: Transforming a square into a torus

when they are combined together. To analyze the incentives to achieve compatibility between the two components, we adopt a variation of Matutes and Regibeau (1988) that accommodates more than 2 varieties in each component market while maintaining symmetry among varieties. There are $n(\geq 2)$ firms, each of which produces the two components of a system, i.e., a single variety of component $A$ and a single variety of component $B .{ }^{6}$ To maintain symmetry, we adopt a torus to represent the product space. Consumers have heterogenous preferences over the characteristics of each component. Each consumer's preference is summarized by her location which represents her ideal variety $\left(x_{A}, x_{B}\right)$ in the product space. The torus is constructed by the following equivalence relations over $\mathbb{R}^{2}$.

$$
\left(x_{A}, x_{B}\right) \sim\left(x_{A}+1, x_{B}\right) \sim\left(x_{A}, x_{B}+1\right) \text { for any }\left(x_{A}, x_{B}\right) \in \mathbb{R}^{2}
$$

As shown in Figure 1, the torus is homeomorphic to the Cartesian product of two circles, i.e. $S^{1} \times S^{1}$, thus is a natural two-dimensional extension of the circular city model à la Salop (1979).

With $n(>2)$ manufacturers for each component, we can imagine a plethora of possibilities to the extent of which the component products are compatible with each other, most of which are analytically intractable. Here, we just consider two polar cases. In the first case, which we simply label as compatibility, any component $A$ is compatible with any arbitrary component $B$ to make a feasible system. Under compatibility, $n^{2}$ systems are available in the market, which is the the maximum number of varieties that can be assembled by the consumers. This case arises if all manufacturers participate in a standard-setting organization, and establish industry-wide standards that would allow "mix-and-match" between two components from any producers. In the other extreme case of compatibility, which we call incompatibility, a brand of component $A$ can be matched only with the component

\footnotetext{
${ }^{6}$ In section 4, we also consider the case where each firm produces only one of the components, and show that our main result is strengthened in this alternative market structure.
} 
$B$ by the same producer. ${ }^{7}$ Incompatibility among different systems prevails if the firms decide to make their components incompatible with their rivals'. Therefore, under incompatibility, only $n$ system goods, the least possible number of varieties, are available to the consumers. Let us denote the set of component $A$ by $I_{A}$, and similarly for $B$ by $I_{B}$. Also let $I_{C}$ be the set of system goods available in the market, and $(i, j)$ be its generic element where $i \in I_{A}$ and $j \in I_{B}$. The size of $I_{C}$ is $n^{2}$ under compatibility, and $n$ under incompatibility. As in Salop (1979), we assume that all varieties are symmetrically located, and focus on symmetric equilibria in which firms independently set the price of their own components, taking the compatibility configurations and location choices as given.

Consumers are uniformly distributed on the torus. A consumer, who is at $\left(x_{A}^{o}, x_{B}^{o}\right)$ and purchases system $(i, j) \in I_{C}$, derives net utility of

$$
v-t\left[\left(x_{A}^{o}-x_{i}\right)^{2}+\left(x_{B}^{o}-x_{j}\right)^{2}\right]-p_{i}^{A}-p_{j}^{B},
$$

where $v$ is the reservation value of the ideal system, which is common to all consumers, $t>0$ is a "transportation cost" parameter that represents the degree of product differentiation, $x_{i}$ is the location of firm $i$ on coordinate $A, p_{i}^{A}$ is the price of component $A$ produced by firm $i$, and $x_{j}$ and $p_{j}^{B}$ are defined similarly for component $B .^{8} \quad$ Each consumer buys at most one unit of the system good that provides the highest net utility. We assume that $v$ is sufficiently large, and thus every consumer makes a purchase in any equilibrium. Each firm's marginal cost is normalized to zero.

\section{Market Equilibrium: Compatibility vs. Incompatibility}

In this section, we derive the market equilibrium under compatibility and incompatibility to analyze incentives to achieve industry-wide standardization. Matutes and Regibeau (1988) address the same question for the case of $n=2$, and show the alignment of private and social incentives for compatibility. We demonstrate that their results are limited to the special case of two firms and not robust to changes in the number of firms. ${ }^{9}$

\footnotetext{
${ }^{7}$ Alternatively, each firm provides an integrated system good of which components cannot be disintegrated and rematched with other products in the market.

${ }^{8}$ We adopt a quadratic transportation cost in our analysis as in d'Aspremont et al. (1979). However, any specification of transportation costs which is a monotonic transformation of the Euclidean distance generates the same qualitative results.

${ }^{9}$ Matutes and Regibeau (1988) also analyze the case where the market is not fully covered.
} 


\subsection{Equilibrium under Compatibility}

Under compatibility, competition takes place at the component level. As a consequence, the symmetric equilibrium is identical to that of the one-dimensional circular city model. This can be easily shown by considering an individual consumer's utility maximization problem:

$$
\begin{array}{r}
\max _{i \in I_{A}, j \in I_{B}}\left\{v-t\left[\left(x_{A}^{o}-x_{i}\right)^{2}+\left(x_{B}^{o}-x_{j}\right)^{2}\right]-p_{i}^{A}-p_{j}^{B}\right\} \\
=v-\min _{i \in I_{A}}\left\{t\left(x_{A}^{o}-x_{i}\right)^{2}+p_{i}^{A}\right\}-\min _{j \in I_{B}}\left\{t\left(x_{B}^{o}-x_{j}\right)^{2}+p_{j}^{B}\right\}
\end{array}
$$

In words, with compatibility that allows every component in $A$ to be combined with any component in $B$, the choice of each component can be made independently of the other. Furthermore, each marginal distribution of consumers' preferences is uniform on a circle of length 1 , since the preferences for $A$ and $B$ are jointly uniform on the torus. Therefore, provided that the products in $I_{A}$ are equidistant from each other along the (say, horizontal) coordinate $x_{A}$ and those in $I_{B}$ along the (vertical) coordinate $x_{B}$, there exists a symmetric equilibrium where all firms in the market set their prices the same and share the market equally.

Note that every symmetric formation of the products on a circle yields the same intensity of competition, which yields the same level of equilibrium profits. As shown in the next subsection, however, this is not the case on the torus: there is usually more than one way to symmetrically locate the products on the torus, and each configuration yields a different level of equilibrium profits.

Since the component market $B$ is structurally identical to market $A$, we just consider the market for component $A$ (horizontal circle) below. Suppose firm $i$, located at the origin, charges $p_{i}^{A}$, and all the other firms charge the identical price $p$ for their component $A$. A consumer located at $x$ is indifferent between purchasing from firm $i$ and purchasing from $i$ 's closest neighbor if $p_{i}^{A}-t x^{2}=p+t\left(\frac{1}{n}-x\right)^{2}$. There are two neighboring firms on the circle. Thus, the demand for firm $i$ 's component $A$ can be written as

$$
D_{i}\left(p_{i}^{A}, p\right)=2 x=\frac{1}{n}-\frac{n\left(p_{i}^{A}-p\right)}{t},
$$

and the profit from component $A$ as

$$
p_{i}^{A} D_{i}\left(p_{i}^{A}, p\right)=p_{i}^{A}\left[\frac{1}{n}-\frac{n\left(p_{i}^{A}-p\right)}{t}\right] .
$$

Differentiating with respect to $p_{i}^{A}$ and imposing the symmetry condition that $p_{i}^{A}=p$, we derive that the equilibrium price and the profit from component $A$ are $t / n^{2}$ and 

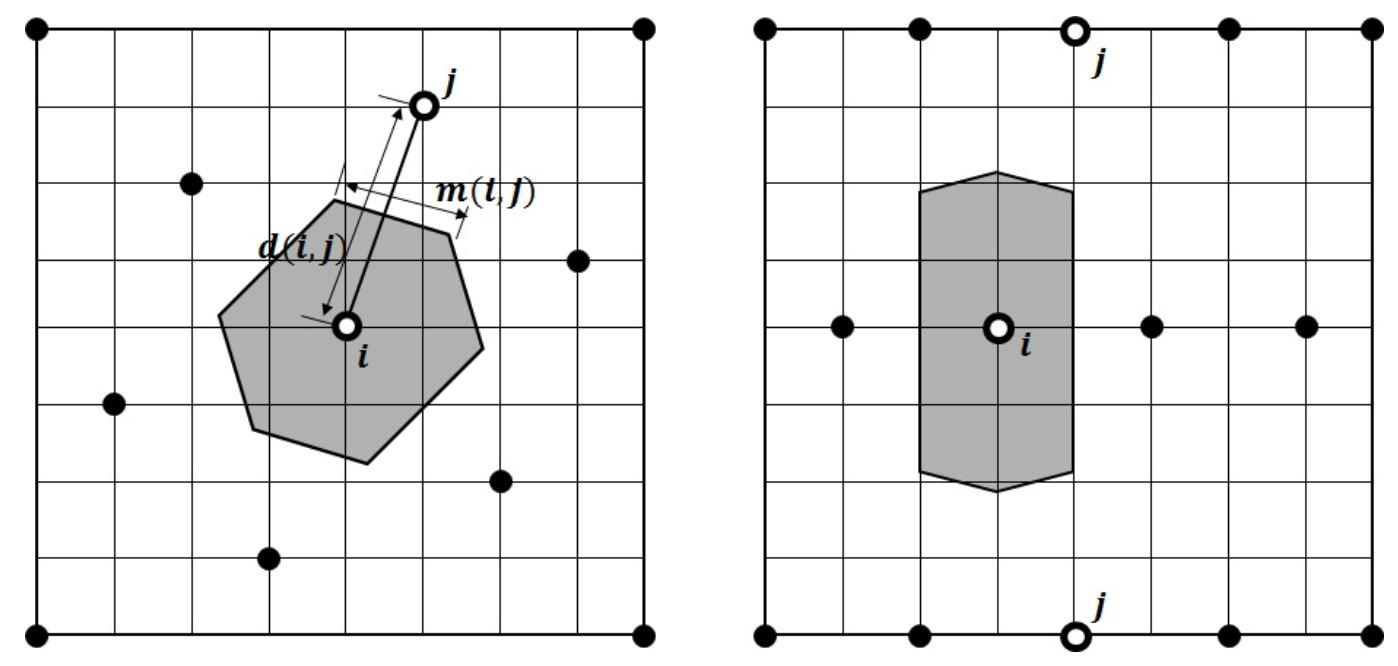

Figure 2: Two symmetric formations with $n=8$

$t / n^{3}$, respectively . Since a firm collects profits from both the $A$ and $B$ markets, the total profit is twice as large as the profit from a component market.

Proposition 1. Under compatibility, the symmetric equilibrium price and profit are given by $p^{C}(n)=t / n^{2}$ and $\pi^{C}(n)=2 t / n^{3}$, respectively.

\subsection{Equilibrium under Incompatibility}

Suppose now that each firm produces the components of a system that are incompatible with the other firms' products. Since the number of available systems under incompatibility is $n$ as opposed to $n^{2}$ (the number under compatibility), and because each firm supplies a single good (i.e., an integrated system), our model is identical to a two-dimensional circular city model with $n$ identical firms. In what follows, with a slight abuse of notation, we use the same notation for the firms and the systems that they produce. As mentioned before, we focus on symmetric equilibria, but there is in general more than one way to symmetrically locate the products on the torus. Formally, we define a symmetric formation as a distribution of the feasible systems on the torus, with which given the same system prices, the shape and size of market areas are identical across the systems. Let $\digamma_{n}$ be the set of all symmetric formations for given $n$, and $F_{n}$ be a generic element of it.

Figure 2 shows two examples of symmetric formations for $n=8$, which yields different equilibrium prices and profits. Note that all dots at the corners of the square in the left panel represent the same system because $\left(x_{A}, x_{B}\right) \sim\left(x_{A}+1, x_{B}\right) \sim$ $\left(x_{A}, x_{B}+1\right)$. By the same token, in the right panel the dots on the top boundary 
and the corresponding dots at the bottom represent the same systems. The shaded areas represent the consumers purchasing one unit of goods from firm $i$, i.e., firm $i$ 's market areas.

Let $N_{i}$ be the set of the neighboring systems of system $i$, where the neighboring systems are defined as follows. In a symmetric equilibrium, $j$ is a neighboring system of $i$ if there exists a set of consumers who are indifferent between $i$ and $j$ and purchase either of the systems. In other words, the neighboring systems are direct competitors who share a market boundary. As in the circular city model where a firm has two direct competitors, in our torus city model, a system competes directly against neighboring systems.

To characterize the equilibrium price and profit for any symmetric formation $F_{n}$, let us introduce a few more definitions. Let $m\left(i, j ; F_{n}\right)$ denote the length of the equilibrium market boundary between system $i$ and its neighboring system $j$, and let $d\left(i, j ; F_{n}\right)$ be the Euclidean distance between these two systems on the torus under symmetric formation $F_{n}$. Using this notation, we define the $m$-to- $d$ ratio, $\mu\left(F_{n}\right)$ for an arbitrary firm $i$,

$$
\mu\left(F_{n}\right)=\sum_{j \in N_{i}} \frac{m\left(i, j ; F_{n}\right)}{d\left(i, j ; F_{n}\right)}
$$

which is the sum of the ratios of two orthogonal segments (see the left panel of Figure 2 ). The following proposition shows how this ratio relates to the equilibrium prices and profits.

Proposition 2. Given a symmetric formation $F_{n}$, the unique symmetric equilibrium price and the corresponding profit under incompatibility are given by $p^{I N}\left(F_{n}\right)=$ $2 t /\left[n \mu\left(F_{n}\right)\right]$ and $\pi^{I N}\left(F_{n}\right)=2 t /\left[n^{2} \mu\left(F_{n}\right)\right]$, respectively.

Proof. See the Appendix.

Note first of all that the equilibrium price depends on the locational formation of the competing products on the torus. More specifically, the proposition shows that the intensity of price competition in the market is completely characterized by and is inversely related to the $m$-to- $d$ ratio $\mu\left(F_{n}\right)$ and the number of firms $n$. Intuitively, the market boundary $m\left(i, j ; F_{n}\right)$ represents the measure of marginal consumers who would respond to a small price change, meaning that a higher $m\left(i, j ; F_{n}\right)$ would lead the firms to engage in more intense price competition. On the other hand, the distance $d\left(i, j ; F_{n}\right)$ captures the extent of product differentiation between the two competing systems $i$ and $j$. The farther the two systems are located, the less substitutable they become. Proposition 2 essentially states that the (symmetric) equilibrium profits increase as the location configuration induces shorter market boundaries 
and longer distances from each other. This result generalizes the principle of maximum differentiation in a one dimensional location model and is consistent with Irmen and Thisse (1998) who show that firms seek the formation which generates the smallest market boundary. ${ }^{10}$

The result can be further appreciated with the examples in Figure 2. The products in the left panel are distributed more evenly over the space, while those in the right panel are concentrated on two horizontal lines. ${ }^{11}$ Thus, we expect that the price competition will be less severe, and therefore the equilibrium profit will be higher with the formation on the left panel. Using Proposition 2, we can confirm this intuition; the equilibrium profit generated from the formation on the left panel is $t / 112$, which is about $35 \%$ higher than $t / 152$, the profit from the formation on the right.

It is also noteworthy that the $m$-to- $d$ ratio itself has little to do with the number of firms $n$. That is, because the length of a market boundary is scaled by the associated distance between the products, the ratio does not necessarily shrink down to zero as $n$ grows to infinity. Instead, the ratio depends crucially on the shape of the market area; when the systems are distributed more evenly over the product space, the shape of each market area becomes more round, as we can see again in Figure 2. We can predict that when the shape of equilibrium market areas is more round, its $m$-to- $d$ ratio is lower, and thus the equilibrium profit will be higher. ${ }^{12}$

Since the equilibrium profit depends on the locational configuration, unlike in the case of compatibility, it is inevitable to search for the profit-maximizing formations for each $n$. As one can imagine, however, finding the profit-maximizing formation among all symmetric formations is by no means easy. So, instead of characterizing the exact maximum of equilibrium profits for each $n$, we construct an upper bound on the minimum $m$-to- $d$ ratios for an arbitrary $n$. This upper bound will later be used to establish that for $n \geq 4$, under incompatibility there exists at least one formation that allows a profit higher than the equilibrium profit under compatibility.

To derive an upper bound for the minimum $m$-to- $d$ ratios for a general $n$, we first introduce a class of simple and tractable formations. Given $n$, the $k$-jump formation, denoted by $J_{n}^{k}$, is the formation in which every system product lies equidistantly on

\footnotetext{
${ }^{10}$ They state that "the lower the density of marginal consumers, the lower is the elasticity. Accordingly, as the consumer distribution is uniform, demand has minimal elasticity when the corresponding hyperplane has minimal surface area."

${ }^{11}$ Note again that the top and the bottom boundaries represent the same line in the torus.

${ }^{12}$ If, hypothetically, the market area is completely round (i.e., a disk), its $m$-to- $d$ ratio would be its circumference over its diameter, which must be approximately 3.141592 .
} 

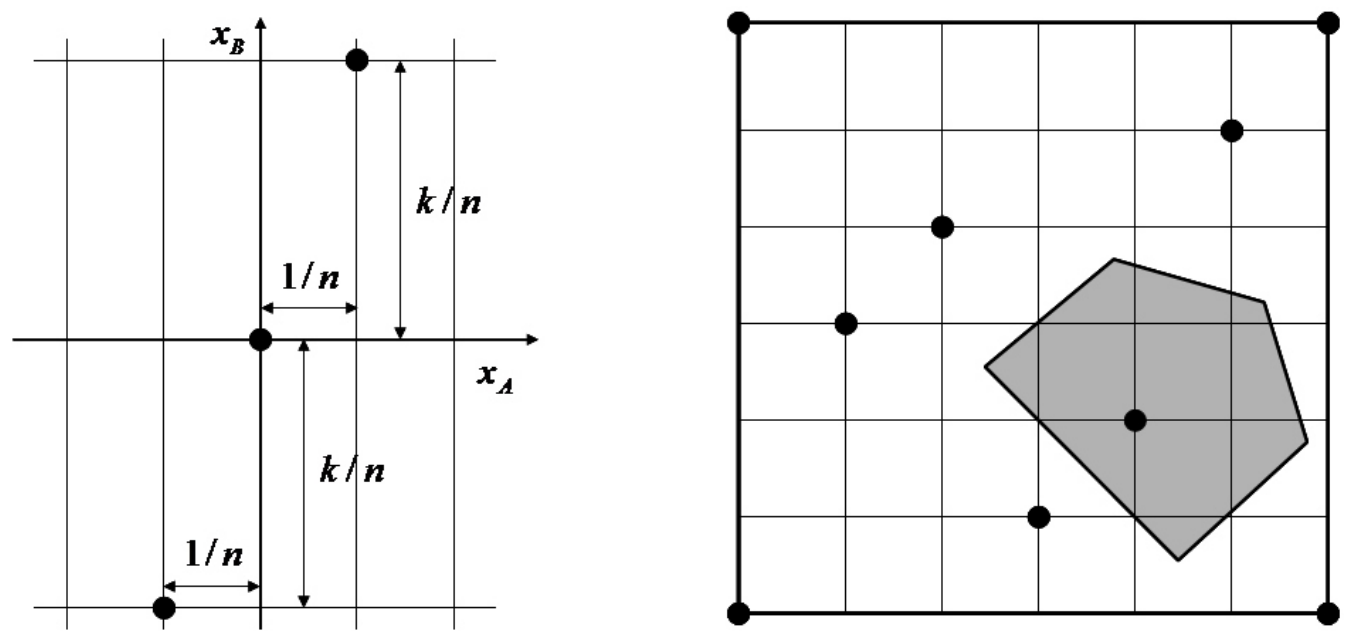

Figure 3: $k$-jump formation (left); a symmetric but not $k$-jump formation (right)

the line of slope $k$ (with intercept zero) for some integer $k$ (see the left panel of Figure 3). ${ }^{13}$ It is noteworthy that the examples in Figure 2 are $k$-jump formations with $n=8$ and $k=3$ and 4 , respectively. A $k$-jump formation is well defined for any $n$ and $k$, and is tractable because it is repetitive.

Note that there are formations which are symmetric but not $k$-jump. The right panel of Figure 3 provides such an example. To make a contrast between $k$-jump formations and the one in the right panel of Figure 3, we introduce another definition that helps categorize the shape of market areas. Suppose that all firms charge the same price while the market is fully covered. A regularly symmetric formation is a symmetric formation in which (i) the number of the market boundaries of each system is an even number, and (ii) for any parallel market boundaries of a system, the distance from a market boundary to the system is the same as the distance from any other boundary to the system. In other words, a regularly symmetric formation has each system locate at the center of its market area. Then, it is apparent that the formation depicted in the right panel of Figure 3 is an example of a symmetric but not regularly symmetric formation. Below, we focus on regularly symmetric formations, and let $F_{n}^{*}$ denote the profit-maximizing formation among

\footnotetext{
${ }^{13}$ When $k$ is a rational number, a formation can be defined in a similar way. But we do not consider such cases here. If $k$ is an irrational number, the line with slope of $k$ never goes back to an integer point. Thus, there does not exist a regularly symmetric formation in which systems are located on a line with slope of an irrational number.
} 


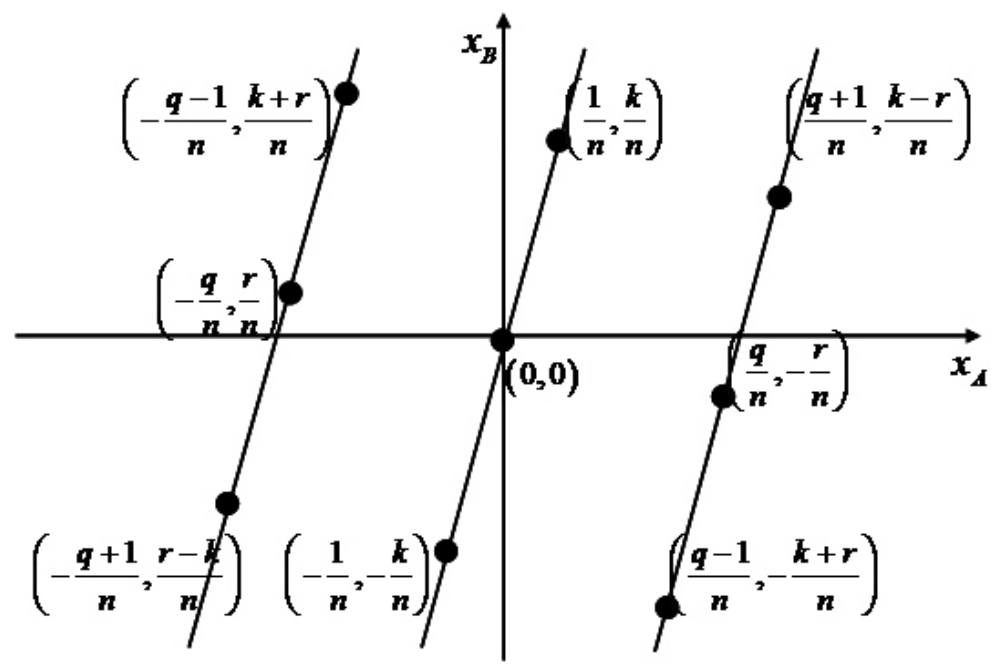

Figure 4: The candidates for neighboring systems of the system at the origin in $k$-jump formation for $k \geq 2 ; q$ is the quotient of the division of $n$ by $k$, and $r$ is the remainder, i.e., $n=q k+r$.

regularly symmetric ones. ${ }^{14}$

The following lemma states some useful facts about $k$-jump formations.

Lemma 1. For any natural number $k$, the following are true:

(i) $k$-jump formations are regularly symmetric.

(ii) With a k-jump formation, the shape of an equilibrium market area is either a rectangle or a hexagon.

Proof. See the Appendix

Note that there exist regularly symmetric formations that are not $k$-jump. For instance, consider a formation where each system is located at the nodes of a square grid. This formation is well defined for any square number $n$, i.e., $n=a^{2}$ for some integer $a \geq 2$, and is not a $k$-jump formation because there does not exist a single line on which all products are located. This, together with the lemma, implies that

\footnotetext{
${ }^{14}$ We restrict our attention to regularly symmetric formations for two reasons. First, because the irregular formations are, as the name suggests, irregular, full characterization of all possible irregular formations is often intractable for large $n$. More importantly, although it is difficult to show this formally, it is intuitively apparent that if a formation locates each system more closely to one market boundary than the others, the formation is not the profit-maximizing one. Therefore, we strongly believe that the set of profit-maximizing formations is a subset of that of regularly symmetric formations.
} 
the set of $k$-jump formations is a subset of that of regularly symmetric formations in general, and thus $k$-jump formations do not help us characterize a lower bound of the $m$-to- $d$ ratio. But it can still help provide an upper bound of the minimum $m$-to- $d$ ratio. Specifically, we construct an upper bound of the minimum $m$-to- $d$ ratio by considering $k$-jump formations, with $k$ the largest integer that is smaller than or equal to $\sqrt{n}$.

Lemma 2. For any $n \geq 2$, the $m$-to-d ratio of the profit-maximizing formation $\mu\left(F_{n}^{*}\right)$ must be smaller than $3 \sqrt{13}$.

Proof. See the Appendix.

\subsection{Comparison between the Regimes}

In this section, we compare equilibrium profits under compatibility and incompatibility. Matutes and Regibeau (1988) show that the profits under compatibility are higher than those under incompatibility when $n=2 .{ }^{15}$ However, we show that their conclusion is overturned when $n \geq 4$.

The upper bound established in Lemma 2 is simply too loose to allow us to show that the equilibrium profits under incompatibility are greater than those under compatibility. But as shown in Propositions 1 and 2, the equilibrium profit under compatibility converges to zero at a rate of $n^{3}$ while the rate of convergence under incompatibility is $n^{2}$, provided that $\mu\left(F_{n}^{*}\right)$ is bounded above. Therefore, incompatibility must be preferred by the firms for sufficiently large $n$. It turns out that such $n$ is not very large. Figure 5 shows the increasing trend of $\pi^{I N}\left(J_{n}^{k^{*}}\right) / \pi^{C}(n)$ where $J_{n}^{k^{*}}$ is the profit-maximizing formation among all $k$-jump formations.

Proposition 3. For any $n \geq 4$, there exists at least one symmetric formation under incompatibility, which allows higher equilibrium profits than those under compatibility.

Proof. See the Appendix.

This result can be appreciated through the lens of Proposition 2, which states that the equilibrium profit is inversely related to the $m$-to- $d$ ratio. Under compatibility,

\footnotetext{
${ }^{15}$ As in our paper, the main analysis of Matutes and Regibeau (1988) focuses on two integrated firms that offer both components, but they point out that the profits are independent of the compatibility regime if the two integrated firms are replaced by four independent single component producers. In section 4, we consider the case of vertically separated firms that offer only one component.
} 


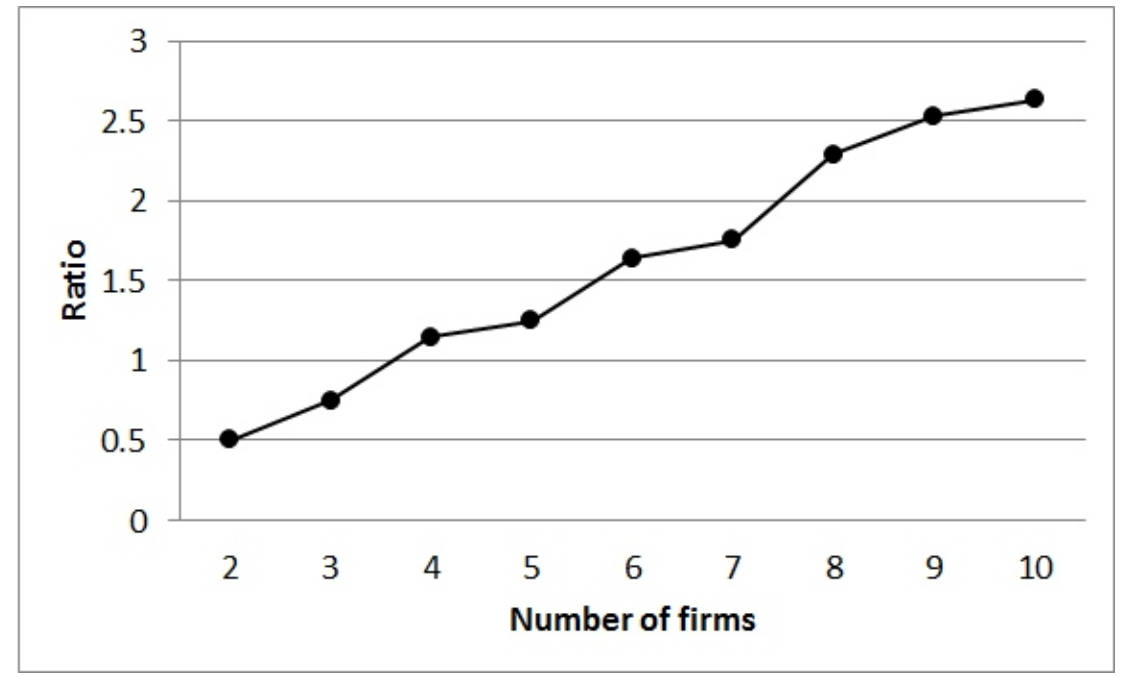

Figure 5: Equilibrium profit ratio $\pi^{I N}\left(J_{n}^{k^{*}}\right) / \pi^{C}(n)$ as a function of $n$

the competition is at the component level, and firm $i$ has two direct competitors, with each of whom the firm shares a market boundary of length 1 . Since the distance between two systems is $1 / n$, the $m$-to- $d$ ratio under compatibility is $2 n$. Note that this specific $m$-to- $d$ ratio can be generated under incompatibility as well by what can be called a 0-jump or no jump formation in which all feasible systems are restricted to lie on a horizontal line. However, firms can do much better than this under incompatibility by scattering their products more evenly over the characteristics space. As shown above, there are configurations which shorten the market boundaries and differentiate the systems more, which in turn softens price competition and increases profits.

In Matutes and Regibeau (1988), the main difference between compatibility and incompatibility in terms of price competition is the degree to which firms can appropriate the benefit of a price reduction; under compatibility, a reduction in the price of component $A$ produced by firm $i$ will increase the market share not only of the system produced by firm $i$, but also of the system of component $A$ produced by firm $i$ and component $B$ produced by its rival. In contrast, under incompatibility the benefit of a price cut is fully captured by the firm. Therefore, price competition is more intense under incompatibility, so firms prefer compatibility to incompatibility. In our model, another effect of compatibility manifests itself more clearly as the number of firms grows larger. More systems are feasible under compatibility $\left(n^{2}\right)$ than under incompatibility $(n)$, which makes the market more crowded and the systems less differentiated from each other. This effect grows larger as the number 
of firms increases, and eventually dominates the effect demonstrated by Matutes and Regibeau.

It is worth mentioning that for $n=2$, our model generates the same result as in Matutes and Regibeau (1988), i.e., the equilibrium profit under compatibility is twice as large as that under incompatibility: $\pi^{C}(2)=t / 4$ while $\pi^{I N}\left(J_{2}^{1}\right)=t / 8 .^{16}$ Our framework thus replicates the result in Matutes and Regibeau (1998) for the duopoly case. However, we show that their result is reversed when the number of firms is equal to or exceeds 4 as the product differentiation effect eventually dominates the "internalization of price-cutting benefits" effect with an increase of $n$. A recent working paper by Zhou (2014) derives a similar result to ours in the context of pure bundling even though he adopts the random utility framework of Perloff and Salop (1985) in contrast to our spatial competition model. He analyzes incentives for bundling with an arbitrary number of firms and shows that under fairly general conditions, bundling raises market prices compared to separate sales when the number of firms exceeds a threshold level. Bundling and separate sales in his model can be reinterpreted as incompatibility and compatibility across components produced by different firms. This confirms that our main result is robust and not model-specific.

The discussion so far suggests that incompatibility, which provides the smallest number of varieties, is most likely to be the profit-maximizing compatibility configuration among all possible compatibility regimes, including the ones not considered in this paper. This is because as more systems are added to the market, a system is likely to encounter more neighbors (i.e., directly competing systems) at nearer locations. In contrast, compatibility can be regarded as the welfare-maximizing form of compatibility when the number of firms is fixed: given the assumption that every consumer purchases a system good, the social welfare is completely determined by the total "transportation costs," which is minimized when the number of available systems is maximized.

\section{Vertically Separated Producers}

Section 3 showed that for $n$ large enough (i.e., $n \geq 4$ ), incompatibility yields higher equilibrium profits than compatibility when each firm produces both component $A$ and component $B$. In this section, we consider an alternative market

\footnotetext{
${ }^{16}$ When the market is served by vertically separated producers as assumed in Section 4 , the firms earn the identical profits $(t / 8)$ under both regimes, which is also the case in Matutes and Regibeau (1988).
} 
structure, following Matutes and Regibeau (1988), in which each component producer is an independent entity, and show that the main result derived above still holds in this alternative market structure.

Matutes and Regibeau (1988) analyze compatibility incentives for two different market structures. In one case, as in the model considered so far, a component $A$ producer is vertically integrated with a component $B$ producer from the beginning. In the other case, each component producer is assumed to be an independent entity, and has to form an alliance with a partner company to make their products compatible. The integrated system providers set their component prices to maximize the profits from both components, internalizing the effect of a price change in one component on the sales of the other. In contrast, the vertically separated component providers do not internalize such externalities. As explained at the end of the previous section, an important effect of compatibility configuration is its effect on the degree of internalization of price externalities. This effect, however, is absent in a market with independent component producers because the firms do not internalize the externalities under both compatibility and incompatibility. Thus, it is not surprising that the key result in Section 3 reappears in a stronger form.

Proposition 4. Suppose both components of the system are provided by vertically independent firms. Then, for any $n \geq 3$, there exists at least one symmetric formation under incompatibility, which allows higher equilibrium profits than those under compatibility.

Proof. See the Appendix.

This proposition is immediate from noticing that individual firms' first order conditions are mathematically identical to the ones derived in the previous analysis. This implies that the equilibrium component price under incompatibility is the same as the equilibrium system price with integrated producers, whereas the prices remain the same under compatibility. As a result, the system price under incompatibility is doubled due to the change in the vertical structure. This is because under incompatibility each component producer ignores the effect of its price cut on the partner firm's demand, which results in double marginalization of system prices. However, this is beneficial to producers in competitive environments because it relaxes price competition as in Bonanno and Vickers (1988).

\section{Concluding Remarks}

In this paper, we examine private and social incentives for compatibility in a twodimensional spatial competition model of system markets. We show that there is a 
fundamental discrepancy between private and social incentives towards compatibility among different vendors. Consumers find compatibility more attractive because it allows more variety of systems, and they can easily find a system close to their ideal specification on average. However, the availability of more variety under compatibility implies intensified competition and a lower profit for every firm in the market. If the number of firms in a system market is fixed, then firms prefer incompatibility to compatibility. However, social welfare is higher under compatibility.

We analyze the private incentives to achieve compatibility assuming the number of firms in the industry is fixed. In the previous version of this paper, we have also performed a long-run analysis in which the number of firms is endogenously determined by the zero profit condition, assuming that the components are provided by vertically independent firms. ${ }^{17}$ We can show that more firms enter under the incompatibility regime due to higher profits compared to under the compatibility regime. $^{18}$ Nonetheless, the number of available systems is still higher under compatibility. This implies that compatibility is the optimal regime in the long-run as well as in the short run because it provides more variety with less entry fixed costs.

We have not explicitly considered any costs involved in achieving a particular form of compatibility. If there are differences in such costs across regimes, the attractiveness of each regime would change in a predictable way. However, it is not clear a priori which type of compatibility would be more costly. In cases where the cost of forming an exclusive coalition (e.g., transaction costs involved in signing a contract) is non-negligibly higher than the cost of establishing standards that would ensure interoperability across all manufacturers, the firms may find compatibility more attractive. However, in other cases the cost of achieving compatibility may be higher than that of incompatibility. ${ }^{19}$

One shortcoming of our paper is that we consider only two possible compatibility regimes: compatibility and incompatibility. For the case of incompatibility, we have not considered each firm's incentive to deviate and build an alliance with another firm. Suppose that every firm in the market decides to make their com-

\footnotetext{
${ }^{17}$ The details of the analysis are available from the authors.

${ }^{18}$ With vertically integrated firms, this result needs to be somewhat weakened because profits under the compatibility regime is higher than profits under the incompatibility regime when $n<4$. However, we can state that there is a threshold value of fixed entry costs $\bar{K}$ such that $n_{C} \leq n_{I N}$ if $K \leq \bar{K}$, where $n_{C}$ and $n_{I N}$ are the number of firms in the free-entry equilibrium under compatibility and incompatibility, respectively, and $K$ denotes entry costs.

${ }^{19}$ In the context of exclusive dealing, Besanko and Perry (1994) argue that exclusive dealing incompatibility in the context of our model - reduces the retailing cost such as the costs of inventory and store space.
} 
ponents incompatible with its rivals' products. Firm $i$ may then have an incentive to approach $j$ and offer to build a new system $(i, j)$ by making their components compatible. If firm $j$ accepts the offer, now there are two feasible systems in the market whose component $A$ is made by firm $i$. Even though the firm $i$ may earn a smaller profit from its own integrated system $(i, i)$, the loss may be more than made up by an additional source of revenue from system $(i, j)$. A full analysis of endogenous formation of coalitions that account for externalities among coalitions in our model would be an important research agenda. ${ }^{20}$ In addition, an industry may be partially compatible, or compatibility may run in only one direction in that some firms' component products can be used in combination with the rival firms', but not the other way around. For instance, Windows OS can be used in Mac computers which allows for dual booting, but Mac OS may not be used with the PC. This type of situation typically arises when firms are asymmetrically situated in the presence of network externalities. ${ }^{21}$ An analysis of partial or one-way compatibility within a general framework of $n$ firms is beyond the scope of the current paper, but would be a worthwhile extension.

\section{Appendix A. Proofs}

Proof of Proposition 2. We first characterize symmetric first order conditions using the $m$-to- $d$ ratio, and then show that the first order condition should be satisfied in equilibrium.

Suppose that given a symmetric formation $F_{n}$, firm $i$ located at $\left(x_{i}^{A}, x_{i}^{B}\right)$ charges $P_{i}$ for its system good while the other firms charge $P$. When $j$ is an element of $N_{i}$, a consumer located at $\left(x_{A}, x_{B}\right)$ is indifferent between system $(i, i)$ and $(j, j)$ if

$$
t\left(x_{A}-x_{i}^{A}\right)^{2}+t\left(x_{B}-x_{i}^{B}\right)^{2}+P_{i}=t\left(x_{A}-x_{j}^{A}\right)^{2}+t\left(x_{B}-x_{j}^{B}\right)^{2}+P
$$

or equivalently,

$2 t\left(x_{j}^{A}-x_{i}^{A}\right) x_{A}+2 t\left(x_{j}^{B}-x_{i}^{B}\right) x_{B}=P-P_{i}+t\left(\left(x_{j}^{A}\right)^{2}-\left(x_{i}^{A}\right)^{2}\right)+t\left(\left(x_{j}^{B}\right)^{2}-\left(x_{i}^{B}\right)^{2}\right)$.

Note that the above formula describes the market boundary that is orthogonal to the line connecting system $i$ and system $j$. To see how much the market boundary moves in the direction of coordinate $A$ as a response to a price change in $P_{i}$, we

\footnotetext{
${ }^{20}$ See Bloch (1996) and Yi (1997) for such an analysis.

${ }^{21}$ For an analysis of one-way compatibility, see Besen and Farrell (1994) and Katz and Shapiro (1985).
} 
first fix $x_{B}$. Then, it is clear that a small increase in $P_{i}$ moves the market boundary along coordinate $A$ as much as $\partial x_{A} / \partial P_{i}=-1 /\left[2 t\left(x_{j}^{A}-x_{i}^{A}\right)\right]$. Now, let us define a new coordinate which is orthogonal to the market boundary, and see how much the boundary moves along this new coordinate. Letting $x_{(i, j)}$ be the projection of $x_{A}$ onto the new coordinate, the following is immediate from the Pythagorean theorem.

$$
\frac{\partial x_{(i, j)}}{\partial x_{A}}=\frac{x_{j}^{A}-x_{i}^{A}}{\sqrt{\left(x_{j}^{A}-x_{i}^{A}\right)^{2}+\left(x_{j}^{B}-x_{i}^{B}\right)^{2}}}=\frac{x_{j}^{A}-x_{i}^{A}}{d(i, j)}
$$

Therefore, a small increase in $P_{i}$ moves the market boundary toward its orthogonal direction as much as

$$
\frac{\partial x_{(i, j)}}{\partial P_{i}}=\frac{\partial x_{(i, j)}}{\partial x_{A}} \frac{\partial x_{A}}{\partial P_{i}}=-\frac{1}{2 t d(i, j)} .
$$

Next, consider the response of the demand $D_{i}\left(P_{i}, P\right)$ to a price change $\Delta P_{i}$ :

$$
\Delta D_{i}\left(P_{i}, P\right) \approx \sum_{j \in N_{i}}\left[m\left(P_{i}, P ;(i, j)\right) \cdot \Delta x_{(i, j)}+\Delta m\left(P_{i}, P ;(i, j)\right) \cdot \Delta x_{(i, j)}\right]
$$

where $m\left(P_{i}, P ;(i, j)\right)$ is the length of the market boundary given the prices $\left(P_{i}, P\right)$, $\Delta x_{(i, j)}$ is the amount that the market boundary moves toward its orthogonal direction, and $\Delta m\left(P_{i}, P ;(i, j)\right)$ is the corresponding change in the market boundary. Notice that when the price change is small, the second term in the square bracket is of second order, and converges to zero faster than the first-order term in the limit. So by dividing by $\Delta P_{i}$ and taking limits on both sides, we obtain the following formula.

$$
\frac{\partial D_{i}\left(P_{i}, P\right)}{\partial P_{i}}=\sum_{j \in N_{i}} m\left(P_{i}, P ;(i, j)\right) \cdot \frac{\partial x_{(i, j)}}{\partial P_{i}}=-\sum_{j \in N_{i}} \frac{m\left(P_{i}, P ;(i, j)\right)}{2 t d(i, j)}
$$

On the other hand, the first order condition for firm $i$ is

$$
P_{i} \frac{\partial D_{i}\left(P_{i}, P\right)}{\partial P_{i}}+D_{i}\left(P_{i}, P\right)=0
$$

which can be rewritten after imposing the symmetry condition $P_{i}=P=P^{I N}$ as

$$
P^{I N}=-\left.\frac{D_{i}\left(P_{i}, P\right)}{\partial D_{i}\left(P_{i}, P\right) / \partial P_{i}}\right|_{P_{i}=P=P^{I N}}=\frac{1}{n}\left(\sum_{j \in N_{i}} \frac{m(i, j)}{2 t d(i, j)}\right)^{-1}=\frac{2 t}{n \mu\left(F_{n}\right)} .
$$


The corresponding equilibrium profit is $\pi^{I N}\left(F_{n}\right)=2 t /\left[n^{2} \mu\left(F_{n}\right)\right]$.

Let us turn our attention to the existence of equilibrium. If firm $i$ sets $P_{i}=0$, its demand $D_{i}\left(P_{i}, P\right)$ must be positive because $P$ cannot be negative. By continuity of the demand function, for small enough $P_{i}$ the profit must be positive. Once again, by the continuity and boundedness of $D_{i}\left(P_{i}, P\right)$ along with the fact that the profit is zero when $P_{i}>v$, the profit function has non-zero maximum with the best response $P_{i}(P) \in(0, v]$. Because all firms' profit functions are identical and continuous, the best response correspondences are continuous and symmetric. Therefore, there exists at least one pure strategy symmetric equilibrium.

In the remainder of the proof, we show that there does not exist a local maximum at which the profit function is not differentiable. This implies that the strategy profile characterized above is the unique symmetric equilibrium because it is the only symmetric solution of the first order conditions.

To prove the claim, we show that the demand function is convex in $P_{i}$. To this end, we first prove that the market area is convex, i.e., if consumers located at $\left(x_{A}, x_{B}\right)$ and $\left(x_{A}^{\prime}, x_{B}^{\prime}\right)$ purchase system $(i, i)$, then a consumer located at $\left(\alpha x_{A}+(1-\right.$ $\left.\alpha) x_{A}^{\prime}, \alpha x_{B}+(1-\alpha) x_{B}^{\prime}\right)$ for any $\alpha \in[0,1]$ also purchases the same system. Suppose both consumers at $\left(x_{A}, x_{B}\right)$ and $\left(x_{A}^{\prime}, x_{B}^{\prime}\right)$ buy system $(i, i)$. For all $(j, j) \in I_{C} \backslash\{(i, i)\}$, the following inequalities are true.

$$
\begin{aligned}
& v-t\left[\left(x_{A}-x_{i}^{A}\right)^{2}+\left(x_{B}-x_{i}^{B}\right)^{2}\right]-P_{i} \geq v-t\left[\left(x_{A}-x_{j}^{A}\right)^{2}+\left(x_{B}-x_{j}^{B}\right)^{2}\right]-P \\
& v-t\left[\left(x_{A}^{\prime}-x_{i}^{A}\right)^{2}+\left(x_{B}^{\prime}-x_{i}^{B}\right)^{2}\right]-P_{i} \geq v-t\left[\left(x_{A}^{\prime}-x_{j}^{A}\right)^{2}+\left(x_{B}^{\prime}-x_{j}^{B}\right)^{2}\right]-P
\end{aligned}
$$

Rearranging terms, the conditions can be rewritten as

$$
\begin{aligned}
& \left(P-P_{i}\right) / t \geq\left(x_{i}^{A}-x_{j}^{A}\right)\left(2 x_{A}-x_{j}^{A}-x_{i}^{A}\right)+\left(x_{i}^{B}-x_{j}^{B}\right)\left(2 x_{B}-x_{j}^{B}-x_{i}^{B}\right) \\
& \left(P-P_{i}\right) / t \geq\left(x_{i}^{A}-x_{j}^{A}\right)\left(2 x_{A}^{\prime}-x_{j}^{A}-x_{i}^{A}\right)+\left(x_{i}^{B}-x_{j}^{B}\right)\left(2 x_{B}^{\prime}-x_{j}^{B}-x_{i}^{B}\right) .
\end{aligned}
$$

Summing up the above inequalities after multiplying by $\alpha$ and $(1-\alpha)$ respectively, we have

$\left(P-P_{i}\right) / t \geq\left(x_{i}^{A}-x_{j}^{A}\right)\left[2\left(\alpha x_{A}+(1-\alpha) x_{A}^{\prime}\right)-x_{g}-x_{i}\right]+\left(x_{j}-x_{h}\right)\left[2\left(\alpha x_{B}+(1-\alpha) x_{B}^{\prime}\right)-x_{h}-x_{j}\right]$,

which implies that $(i, i)$ is the best choice for any consumer located on the segment connecting $\left(x_{A}, x_{B}\right)$ and $\left(x_{A}^{\prime}, x_{B}^{\prime}\right)$. Therefore, the total length of the market boundaries is monotonically decreasing as $P_{i}$ increases. This, in turn, implies the derivative of the demand $\left(\partial D_{i}\left(P_{i}, P\right) / \partial P_{i}\right)$ is increasing (see equation $($ A.2 $\left.)\right)$ in $P_{i}$, i.e., the demand function is convex in $P_{i}$. Note that there does not exist a non-differentiable 
local maximum of $P_{i} D\left(P_{i}\right)$ if $D\left(P_{i}\right)$ is a convex function. Therefore, in the unique symmetric equilibrium, the first order condition derived above should hold.

Proof of Lemma 1. (i) By definition, there is a line of slope $k$ on which all the products in $I_{C}$ lie. Let us call this line $L$. In $\mathbb{R}^{2}$ representation, infinitely many $L$ 's lie equidistantly from each other by the property of $\left(x_{A}, x_{B}\right) \sim\left(x_{A}+1, x_{B}\right) \sim$ $\left(x_{A}, x_{B}+1\right)$. Furthermore, since the systems are distributed regularly on each line, if a product finds a competing product on one side, it must find another on the exactly opposite side. This means that if we draw a line connecting any two systems and beyond, we would encounter a third system, and the distance between the first and the second is the same as that between the second and the third. See Figure 4 for a concrete picture.

(ii) By result (i), the shape of a market area generated by a $k$-jump formation should be either rectangular, hexagonal, octagonal, or $2 a$-gonal for $a \geq 5$. We first show that it is not possible that a market area is shaped as an octagon. To see this, suppose that there exists $(n, k)$ such that given $n$, a $k$-jump formation generates an octagon-shaped market area. Since the market area is convex as shown in the proof of Proposition 2, each interior angle of the octagon must be smaller than $180^{\circ}$. Thus, three or more vertices are required to complete one $360^{\circ}$. In addition, in tiling the torus ( $\mathbb{R}^{2}$ plane), each vertex of the octagon should participate only once, and all together eight vertices should make three complete $360^{\circ}$ because the sum of the interior angles is $180 \times(8-2)=1080=360 \times 3$. However, it is easy to see that when we divide the eight vertices into three disjoint sets, there is always a set which has only two or less elements. This contradicts the condition that each interior angle is smaller than $180^{\circ}$. By the same token, for $a \geq 5$, one can check that $2 a$ vertices cannot be distributed into $a-1$ disjoint sets without allowing at least one set to have two or less elements.

Proof of Lemma 2. We characterize an upper bound of $\mu\left(F_{n}^{*}\right)$ using a $k$-jump formation. Note that when $n$ is larger than or equal to 4 , in any $k$-jump formation with $k \geq 2$, eight products nearby the focused product are potential neighbors. (See Figure 4.) Since the number of market boundaries is either four or six by (ii) in Lemma 1, at least two products out of the eight cannot participate in forming the market boundaries.

Let $k_{o}=\lceil\sqrt{n}\rceil$, the largest integer that is smaller than or equal to $\sqrt{n}$, and consider a $k_{o}$-jump formation. Suppose that product $i$ is located at the origin. We then label the product at $\left(1 / n, k_{o} / n\right)$ as the first system and the product at $\left(2 / n, 2 k_{o} / n\right)$ as the second system, and so on. Then, since $k_{o}^{2} \leq n$, or equivalently $\left(k_{o}+1\right) k_{o} / n \leq 1+k_{o} / n$, for the $\left(k_{o}+1\right)^{\text {st }}$ system from the origin which is at $\left(\left(k_{o}+1\right) / n,\left(k_{o}+1\right) k_{o} / n\right)$, the maximum distance from the horizontal axis is 
$k_{o} / n$ because $\left(\left(k_{o}+1\right) / n, 1+k_{o} / n\right) \sim\left(\left(k_{o}+1\right) / n, k_{o} / n\right)$. Note that potentially the $\left(k_{o}-1\right)^{\mathrm{st}}, k_{o}^{\mathrm{th}}$, and $\left(k_{o}+1\right)^{\mathrm{st}}$ system can participate in building a market boundary around $i$. (See Figure 4. In this case, $q=k_{o}$ and $r=n-k_{o}^{2}$.) Thus, the distance between $i$ and a neighboring system of $i$ cannot be larger than $\sqrt{\left(k_{o}+1\right)^{2}+k_{o}^{2}} / n$, which is the distance between the origin and $\left(k_{o}+1\right)^{\text {st }}$ system when $k_{o}=\sqrt{n}$. At the same time, it cannot be smaller than $k_{o} / n$, which is the distance from the origin to the $k_{o}^{\text {th }}$ system when $k_{o}=\sqrt{n}$.

For any rectangle, the longest distance between two edges is equal to the length of the longest edge. For any hexagon, the longest distance between two edges is longer than or equal to the longest edge by the triangle inequality. In other words, every market boundary is shorter than or equal to the longest distance between two neighboring systems. Thus, each ratio of a market boundary to the associated distance must be smaller than or equal to $\sqrt{\left(k_{o}+1\right)^{2}+k_{o}^{2}} / n$ over $k_{o} / n$ :

$$
\mu\left(F_{n}^{*}\right) \leq \mu\left(J_{n}^{k_{o}}\right)<6 \cdot \frac{\sqrt{\left(k_{o}+1\right)^{2}+k_{o}^{2}} / n}{k_{o} / n}=\frac{6 \sqrt{2 k_{o}^{2}+2 k_{o}+1}}{k_{o}} \leq 3 \sqrt{13}
$$

where the last inequality comes from the fact that $6 \sqrt{2 k_{o}^{2}+2 k_{o}+1} / k_{o}$ is decreasing in $k_{o}$, and is $3 \sqrt{13}$ when $k_{o}=2$.

For the cases of $n=2$ and 3 , one can easily confirm that $\mu\left(J_{n}^{1}\right)=4<3 \sqrt{13}$.

Proof of Proposition 3. By Lemma 2, the ratio of the equilibrium profit under incompatibility to that under compatibility is larger than $n / 3 \sqrt{13}$, which implies that the ratio is greater than 1 for $n>10$. To complete the proof, we only need to consider the case where $4 \leq n \leq 10$, and show that $\pi^{I N}\left(F_{n}^{*}\right) \geq \pi^{C}(n)$ as follows.

$$
\begin{array}{cc}
\pi^{I N}\left(J_{4}^{2}\right)=t / 28>t / 32=\pi^{C}(4) & \pi^{I N}\left(J_{5}^{2}\right)=t / 50>2 t / 125=\pi^{C}(5) \\
\pi^{I N}\left(J_{6}^{2}\right)=t / 66>t / 108=\pi^{C}(6) & \pi^{I N}\left(J_{7}^{2}\right)=t / 98>2 t / 343=\pi^{C}(7) \\
\pi^{I N}\left(J_{8}^{3}\right)=t / 112>t / 256=\pi^{C}(8) & \pi^{I N}\left(J_{9}^{3}\right)=t / 144>2 t / 729=\pi^{C}(9) \\
\pi^{I N}\left(J_{10}^{4}\right)=t / 190>t / 500=\pi^{C}(10) &
\end{array}
$$

Proof of Proposition 4. Consider first the case of incompatibility. Suppose that given a symmetric formation $F_{n}$, firm $i$ located at $x_{i}^{A}$ forms a coalition with firm $j$ located at $x_{j}^{B}$, and charges $p_{i}$ for its component good while the other firms (including firm $j$ ) charge $p$. Denote $N_{(i, j)}$ as the set of neighbors of system $(i, j)$. When $(g, h)$ is an element of $N_{(i, j)}$, a consumer located at $\left(x_{A}, x_{B}\right)$ is indifferent between system $(i, j)$ and $(g, h)$ if

$$
t\left(x_{A}-x_{i}^{A}\right)^{2}+t\left(x_{B}-x_{j}^{B}\right)^{2}+p_{i}+p=t\left(x_{A}-x_{g}^{A}\right)^{2}+t\left(x_{B}-x_{h}^{B}\right)^{2}+2 p .
$$


Notice that this condition is virtually identical with equation (A.1), which implies the demand for component $A$ made by firm $i$ would also be identical with $D_{i}\left(p_{i}, p\right)$ derived in the proof of Proposition 2. The first order condition would also be identical. Thus, the equilibrium prices of each component here are exactly the same as those of the system prices charged by vertically integrated firms. On the other hand, under compatibility, the intensity of competition is not affected by the vertical structure of the industry, meaning that the profit for a component producer is half of that for an integrated system producer.

In short, under incompatibility each component producer earns as much as $\pi^{I N}\left(F_{n}\right)$, whereas under compatibility they earn $\pi^{C}(n) / 2$. When $n=3$, the maximum profit under incompatibility is $\pi^{I N}\left(J_{3}^{1}\right)=\pi^{I N}\left(J_{3}^{2}\right)=t / 18$, and under compatibility $\pi^{C}(3) / 2=t / 27$. Therefore, even for $n=3$, there exists a symmetric formation under incompatibility that yields a higher equilibrium profit than that under compatibility.

[1] Armstrong, M., 2006. Competition in two-sided markets. Rand J. Econ. 37, 668-691.

[2] Besen, S. M., Farrell, J., 1994. Choosing how to compete: strategies and tactics in standardization. J. Econ. Perspect. 8, 117-131.

[3] Rochet, J.-C., Tirole, J., 2006. Two-sided markets: a progress report. Rand J. Econ. 37, 645-667.

[4] Besanko, D., Perry, M. K., 1994. Exclusive dealing in a spatial model of retail competition. Int. J. Ind. Org. 12, 297-329.

[5] Bloch, F., 1996. Sequential formation of coalitions in games with externalities and fixed payoff division. Games Econ. Behav. 14, 90-123.

[6] Bonanno, G., Vickers, J., 1988. Vertical separation, J. Ind. Econ. 36, 257-265.

[7] Chen, Y., 1997. Equilibrium product bundling. J. Bus. 70, 85-103.

[8] Choi, J. P., 1996. Preemptive R\&D, rent dissipation, and the "leverage theory." Quart. J. Econ. 111, 1153-1181.

[9] D'Aspremont, C., Jaskold-Gabszewicz, J., Thisse, J.-F., 1979. On Hotelling's stability in competition. Econometrica 47, 1145-50.

[10] Denicolo, V., 2000. Compatibility and bundling with generalist and specialist firms. J. Ind. Econ. 48, 177-188. 
[11] Economides, N., 1989. Desirability of compatibility in the absence of network externalities. Amer. Econ. Rev. 79, 1165-1181.

[12] Farrell, J., Monroe, H., Saloner, G., 1998. The vertical organization of industry: systems competition versus component competition. J. Econ. Manag. Strategy $7,143-182$.

[13] Irmen, A., Thisse, J.-F., 1998. Competition in multi-characteristics spaces: Hotelling was almost right. J. Econ. Theory 78, 76-102.

[14] Katz, M. L., Shapiro, C., 1985. Network externalities, Competition, and Compatibility. Amer. Econ. Rev. 75, 424-440.

[15] Katz, M. L., Shapiro, C., 1994. Systems competition and network effects. J. Econ. Perspect. 8, 93-115.

[16] Kim, S.-H., Choi, J. P., 2014. Optimal compatibility in system markets. Unpublished manuscript, available at https://sites.google.com/site/sanghyunkim46/research.

[17] Massey, W., 1991. A basic course in algebraic topology. Springer, New York.

[18] Matutes, C., Regibeau, P., 1988. "Mix and match": product compatibility without network externalities. Rand J. Econ. 19, 221-234.

[19] Matutes, C., Regibeau, P., 1991. Compatibility without network externalities: the case of $\mathrm{N}$ firms. Unpublished manuscript, Northwestern University.

[20] Matutes, C., Regibeau, P., 1992. Compatibility and bundling of complementary goods in a duopoly. J. Ind. Econ. 40, 37-54.

[21] Monroe, H, 1993. Mix and match compatibility and asymmetric cost. Ph.D. thesis, Oxford University.

[22] Nalebuff, B., 2004. Bundling as an entry barrier. Quart. J. Econ. 119, 159-187.

[23] Perloff, J., Salop, S., 1985. Equilibrium with product differentiation. Rev. Econ. Stud. 52, 107-120.

[24] Salop, S., 1979. Monopolistic competition with outside goods. Bell J. Econ. 10, 141-56. 
[25] Yi, S-S., 1997. Stable coalition structures with externalities. Games Econ. Behav. 20, 201-237.

[26] Zhou, J., 2004. Competitive bundling. Unpublished manuscript, available at https://sites.google.com/site/jidongzhou77/research. 